\title{
FRICTIONAL SLIP BETWEEN A LAYER AND A SUBSTRATE DUE TO A PERIODIC TANGENTIAL SURFACE FORCE
}

\author{
Maria Comninou $\dagger$ and J. R. Barber $\ddagger$ \\ University of Michigan, Ann Arbor, MI 48109, U.S.A.
}

(Received 13 April 1982; in revised form 12 July 1982)

\begin{abstract}
A solution is given for the problem of an elastic layer pressed against an elastic half-plane and subjected to a tangential force varying periodically in time. A loading cycle which initially causes localized slip is followed through unloading and reloading. A limiting load is established below which the steady state of the interface does not involve slip.
\end{abstract}

\section{INTRODUCTION}

The problem of frictional slip and separation between an elastic layer and a half-plane due to concentrated surface loads has been considered in a recent series of papers [1-3]. The layer is pressed against the half-plane by a uniform pressure $p_{0}$, as shown in Fig. 1, and a concentrated load $Q$ is then increased monotonically from zero until slip occurs somewhere at the interface. With a further increase in $Q$, this slip zone extends, and the next stage involves either a second slip zone or else separation in the first slip zone depending on the direction of the force and the coefficient of friction.

From the practical point of view, the most serious consequence of slip between contacting surfaces is the damage of those surfaces due to wear. If the slip is restricted to a region of an otherwise non-slipping interface, significant damage will only occur with repeated loading. In particular, this might arise from a load at a fixed point on the surface which varies sinusoidally with time. A typical example of periodic loading related to the system of Fig. 1, is a machine vibrating due to rotating out of balance masse's and bolted through a fiange to an elastic foundation. Systems of this type are subject to failure known as "fretting corrosion" [4, 5]. The failure is initiated by localized slip at the interface: wear particles are detached and, being trapped between the surfaces, act as abrasives and accelerate the damage. With steel surfaces, these particles consist of iron oxides and the conditions favor accelerated corrosion in the slip region, of ten exacerbated by the infusion of water.

Fretting corrosion can be eliminated by preventing slip-typically by increasing the pressure $p_{0}$ between the surfaces. The required pressure can be estimated from [1-3], since if slip does not occur during the first loading cycle, it will never occur. However, this condition may be too restrictive. Frictional slip can be regarded as inducing stress relief in the same way as plastic flow. After a loading-unloading cycle, it will leave a residual stress field which is favorable to the prevention of slip in subsequent cycles. It is, therefore, possible for slip to occur during the first few cycles of loading, but for the system to "shake down" into a steady state without slip. The closely related problem of shakedown due to subsurface plastic deformation in a halfplane subjected to surface loads has been investigated by Merwin and Johnson [6]. At higher levels of periodic loading (above the "shakedown limit") slip will persist in the steady state, but the extent of the slip zones will probably be smaller than in the first loading cycle.

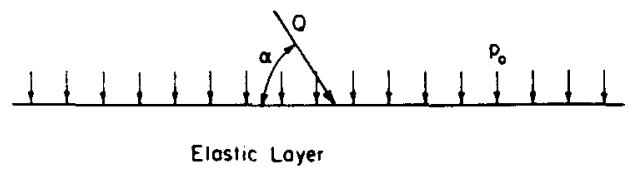

Elostic Half-plone

Fig. 1. Layer-substrate geometry.

tDepartment of Civil Engineering.

$\ddagger$ Department of Mechanical Engineering and Applied Mechanics. 
In this paper we treat the case of periodic loading and establish the shakedown limit for a tangential load, i.e. $\alpha=0$ in Fig. 1. We study the case in which only one slip zone and no separation occurs during the loading phase.

\section{LOADING PHASE}

The loading phase of the problem is identical to the one treated in [3]. However, the formulation is repeated here briefly for reference and use in subsequent sections. The geometry, indicating the first slip zone formed upon loading, is shown in Fig. 2. Higher loads causing two slip zones or separation upon loading will not be considered here.

Before the onset of slip, stick prevails throughout the interface $y=0$ and the Flamant solution [7] is valid. When slip occurs, it is then accounted for by a distribution of glide dislocations with density $B_{x}(x)$ defined on the slip zone $c<x<d$. The total shear $S(x)$ and normal $N(x)$ tractions at the interface are

$$
\begin{gathered}
S(x)=\frac{2 Q}{\pi} \frac{a x^{2}}{\left(a^{2}+x^{2}\right)^{2}}+\frac{2 \mu}{\pi(\kappa+1)}\left\{\int_{c}^{d} \frac{B_{x}(\xi)}{x-\xi} \mathrm{d} \xi+\int_{c}^{d} B_{x}(\xi) K_{s}(x, \xi ; a) \mathrm{d} \xi\right\} \\
N(x)=-p_{0}-\frac{2 Q a^{2} x}{\pi\left(a^{2}+x^{2}\right)^{2}}+\frac{2 \mu}{\pi(x+1)} \int_{c}^{d} B_{x}(\xi) K_{n}(x, \xi ; a) \mathrm{d} \xi
\end{gathered}
$$

where

$$
\begin{gathered}
K_{s}(x, \xi ; a)=\frac{x-\xi}{4 a^{2}+(x-\xi)^{2}}\left\{-1+\frac{12 a^{2}}{4 a^{2}+(x-\xi)^{2}}-\frac{64 a^{4}}{\left[4 a^{2}+(x-\xi)^{2}\right]^{2}}\right\} \\
K_{n}(x, \xi ; a)=\frac{8 a^{3}}{\left[4 a^{2}+(x-\xi)^{2}\right]^{2}}\left[-3+\frac{16 a^{2}}{4 a^{2}+(x-\xi)^{2}}\right] .
\end{gathered}
$$

The non-integral terms in (1) and (2) correspond to the Flamant solution and the uniform pressure $p_{0}$. The dislocation density $B_{x}(x)$ is related to the tangential displacement discontinuity or relative shift $h(x)$ by

$$
B_{x}(x)=-\frac{\mathrm{d} h}{\mathrm{~d} x}
$$

The boundary conditions to be satisfied on the interface are

$$
\begin{gathered}
|S(x)|=f|N(x)|, c<x<d \\
N(x)<0, \text { all } x \\
|S(x)|<f|N(x)|, x<c, x>d \\
\operatorname{sgn} S(x)=\operatorname{sgn} \frac{\mathrm{d} h}{\mathrm{~d} t}, c<x<d .
\end{gathered}
$$

The time dependence shown in (9) enters the equations through $Q$, which is assumed to increase

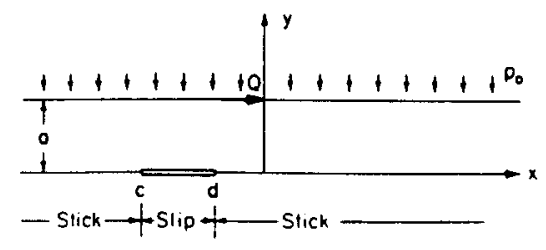

Fig. 2. Geometry of present problem. 


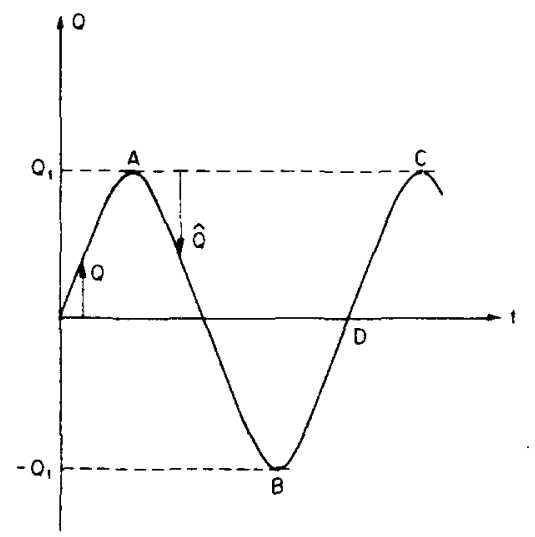

Fig. 3. Variation of load $Q$ as a function of time.

monotonically in time from zero to a maximum value $Q_{1}$ during loading, as shown in Fig. 3. This process is assumed to be sufficiently slow so that inertial effects can be neglected. The Flamant solution suggests that $S(x)$ will remain positive everywhere during loading and hence (6) and (9) can be replaced by (10) and (11)

$$
\begin{gathered}
S(x)=-f N(x), c<x<d \\
h(x)>0 .
\end{gathered}
$$

Equation (7) was taken into account in (10).

In addition to the boundary conditions already described, two further conditions must be satisfied. One concerns uniqueness of displacements and is expressed as

$$
D=\int_{c}^{d} B_{x}(\xi) \mathrm{d} \xi=0
$$

and the other is that $B_{x}(x)$ must be bounded as a consequence of (8), see Ref. [8]. These two conditions provide two extra equations which are needed for the determination of the unknown parameters $c$ and $d$ specifying the slip zone. For further details of the loading solution, the reader is referred to [3].

\section{UNLOADING PHASE}

The tractions corresponding to the maximum load $Q_{1}$ reached during loading will be denoted by $S_{1}(x)$ and $N_{1}(x)$, and the edges of the slip zone by $c_{1}, d_{1}$. Henceforth quantities with subscript 1 refer to the values reached upon loading up to $Q_{1}$. As $Q$ is reduced slightly from $Q_{1}$, (9), and therefore (11), are violated and slip cannot persist in the forward $(h(x)>0)$ direction. Backslip $(h(x)<0)$ cannot start, either, before $Q$ is reduced substantially. Thus, upon unloading stick sets in everywhere and the tractions at the interface are

$$
\begin{aligned}
& S(x)=S_{1}(x)-\frac{2 \hat{Q}}{\pi} \frac{a x^{2}}{\left(a^{2}+x^{2}\right)^{2}} \\
& N(x)=N_{1}(x)+\frac{2 \hat{Q}}{\pi} \frac{a^{2} x}{\left(a^{2}+x^{2}\right)^{2}}
\end{aligned}
$$

where

$$
\hat{Q}=Q_{1}-Q
$$

Upon further decrease in loading backslip eventually starts. During backslip $S(x)$ is negative and (9) requires that

$$
h(x)<0 \text {. }
$$


To allow for the possibility of extending the formulation to cover a number of unloading and reloading cycles, we adopt the following notation

$$
\rho=\left\{\begin{array}{l}
+f \text { for forward slip, or } h(x)>0 \\
-f \text { for backslip, or } h(x)<0
\end{array}\right.
$$

Then (6) and (9) can be replaced by

$$
\begin{gathered}
S(x)=-\rho N(x), c<x<d \\
\operatorname{sgn} h=\operatorname{sgn} \rho
\end{gathered}
$$

which are valid for both forward slip and backslip. We denote the ends of the new slip zone by $(c, d)$. The boundary conditions to be satisfied are (18), (19), (7) and (8). The total tractions to be used in these boundary conditions are obtained by adding to the expressions given by (13) and (14), the integral part of (1) and (3) respectively. The slip zone now appears in a location approximately symmetric to the one shown in Fig. 2 . It is not exactly symmetric because of the influence of the tractions locked-in during the stick stage of unloading.

The presence of the Cauchy integral in (1) restricts the choice of the values of $x$ to be used in the discretization of the problem. For this reason it is preferable to write the equations in terms of locked-in dislocations rather than locked-in tractions. Thus the dislocation distribution reached during loading up to $Q_{1}$ is denoted by $B_{1 x}$ and it is used for the calculation of $S_{1}(x)$ and $N_{1}(x)$.

\section{DISCRETIZATION}

We first normalize the interval $(c, d)$ by the change of variables

$$
u=\delta s+\sigma, \quad v=\delta r+\sigma
$$

where

$$
u=x / a, v=\xi a, \quad \delta=\frac{d-c}{2 a}, \quad \sigma=\frac{d+c}{2 a} .
$$

A similar normalization is made for the interval $\left(c_{1}, d_{1}\right)$. Next we define a regular function $\phi(r)$ by

$$
B_{x}(r)=\lambda \frac{p_{0}(\kappa+1)}{2 \mu}\left(1-r^{2}\right)^{1 / 2} \phi(r)
$$

where $\lambda$ is the dimensionless loading parameter

$$
\lambda=\frac{Q}{p_{0} a}
$$

and $\phi_{1}(r)$ and $\lambda_{1}$ are similarly defined. The total tractions become

$$
\begin{aligned}
S(u) / p_{0}= & \frac{2}{\pi} \lambda \frac{u^{2}}{\left(1+u^{2}\right)^{2}}+\frac{1}{\pi} \lambda_{1} \delta_{1}\left[\int_{-1}^{1} \frac{\left(1-r^{2}\right)^{1 / 2} \phi_{1}(r)}{u-v_{1}} \mathrm{~d} r+\int_{-1}^{1}\left(1-r^{2}\right)^{1 / 2} K_{s}\left(u, v_{1} ; 1\right) \phi_{1}(r) \mathrm{d} r\right] \\
& +\frac{1}{\pi} \lambda \delta\left[\int_{-1}^{1} \frac{\left(1-r^{2}\right)^{1 / 2} \phi(r)}{u-v} \mathrm{~d} r+\int_{-1}^{1}\left(1-r^{2}\right)^{1 / 2} K_{s}(u, v ; 1) \phi(r) \mathrm{d} r\right] \\
N(u) / p_{0}= & -1-\frac{2}{\pi} \lambda \frac{u}{\left(1+u^{2}\right)^{2}}+\frac{1}{\pi} \lambda_{1} \delta_{1} \int_{-1}^{1}\left(1-r^{2}\right)^{1 / 2} K_{n}\left(u, v_{1} ; 1\right) \phi_{1}(r) \mathrm{d} r \\
& +\frac{1}{\pi} \lambda \delta \int_{-1}^{1}\left(1-r^{2}\right)^{1 / 2} K_{n}(u, v ; 1) \phi(r) \mathrm{d} r .
\end{aligned}
$$


For the discretization we use the method of Erdogan and Gupta [9], according to which we choose the integration points $r_{i}$ and collocation points $s_{k}$ of the Cauchy integrals so that

$$
\begin{gathered}
r_{i}=\cos \left(\frac{i \pi}{n+1}\right), i=1, \ldots n \\
s_{k}=\cos \left[\frac{\pi(2 \kappa-1)}{2(n+1)}\right], k=1, \ldots, n+1 .
\end{gathered}
$$

This discretization incorporates the condition of boundedness of $B_{x}(r)$ as an extra equation in the discretized system. For non-Cauchy integrals we use the Chebyshev quadrature and the choice of the collocation points $s_{k}$ is immaterial.

The discretized form of (18) becomes

$$
\begin{aligned}
\lambda \delta \sum_{i=1}^{n} \frac{\left(1-r_{i}^{2}\right)}{n+1}\left[\frac{1}{u_{k}-v_{i}}+K\left(u_{k}, v_{i}\right)\right] \phi\left(r_{i}\right)=\rho-\frac{2}{\pi} \lambda \frac{u_{k}\left(u_{k}-\rho\right)}{\left(1+u_{k}^{2}\right)^{2}} \\
-\lambda_{1} \delta_{1} \sum_{i=1}^{n} \frac{\left(1-r_{i}^{2}\right)}{n+1}\left[\frac{1}{u_{k}-v_{1 i}}+K\left(u_{k}, v_{1 i}\right)\right] \phi_{1}\left(r_{i}\right) \quad k=1, \ldots n+1
\end{aligned}
$$

where

$$
K(u, v)=K_{s}(u, v ; 1)+\rho K_{n}(u, v ; 1) .
$$

The discretized form of (12) is

$$
D=\frac{\lambda p_{0}(\kappa+1) \delta}{2 \mu} \frac{\pi}{n+1} \sum_{i=1}^{n}\left(1-r_{i}^{2}\right) \phi\left(r_{i}\right)=0 .
$$

The system of eqns (18) and (30) is sufficient for the determination of the $n$ values $\phi\left(r_{i}\right)$ and the parameters $c, d$. To simplify the iteration procedure, we interchange the roles of $d$ and $\lambda$. Thus we treat $\lambda$, which appears linearly in (28) as one of the unknowns and $d$ as given. Equation (28) gives then a system of $n+1$ equations for the $n+1$ unknowns $\lambda$ and $\phi\left(r_{i}\right)$, while (30) is used to determine $c$ by iteration. The normal tractions in the stick zones are then computed from (24) and (25). Care must be taken for the evaluation of the shear tractions in the interval $\left(c_{1}, d_{1}\right)$ because of the presence of the Cauchy integral involving $\phi_{1}(r)$. This is done by using the collocation points $u_{1 i}$ in $\left(c_{1}, d_{1}\right)$. The Cauchy-like integral involving $\phi(r)$ is not actually Cauchy in $\left(c_{1}, d_{1}\right)$ because the intervals $\left(c_{1}, d_{1}\right)$ and $(c, d)$ have no common parts.

\section{RESULTS}

In all the numerical computations $f$ was fixed at 0.5 . Figures 4 and 5 show the normal and shear tractions respectively at loading, unloading and reloading for $\lambda_{1}=2.353$. The hooks in the shear tractions correspond to the edges of the past and current slip zones [8]. During the first loading cycle, slip occurs in the range $-1.4445<x / a<-0.4$. and during unloading (to $\lambda=$ -2.353 ), backslip occurs in $0.4039<x / a<1.4391$. Notice that the backslip region is smaller than the original forward slip region. This difference is due to the locked-in dislocations in the original slip zone, which are therefore seen as favorable to preventing slip in this region during unloading. During reloading to $\lambda=2.353$, stick prevails everywhere, and hence subsequent cycles of unloading and loading between these limits will produce no further slip.

Further insight can be gained by considering a loading history in which the first unloading cycle does not proceed far enough to produce backslip. In such a case, if we were to reload past the original maximum load $Q_{1}$, slip would start instantaneously at $Q_{1}$ over the whole of the original slip zone. Now in the case illustrated in Figs. 4 and 5, we find that complete stick prevails past $Q_{1}$, but at a slightly higher load a slip zone starts and spreads rapidly over the original zone. The difference between these two loading histories is due to the locked-in dislocations in the unloading backslip zone and these have a small but beneficial effect in preventing slip on reloading. 

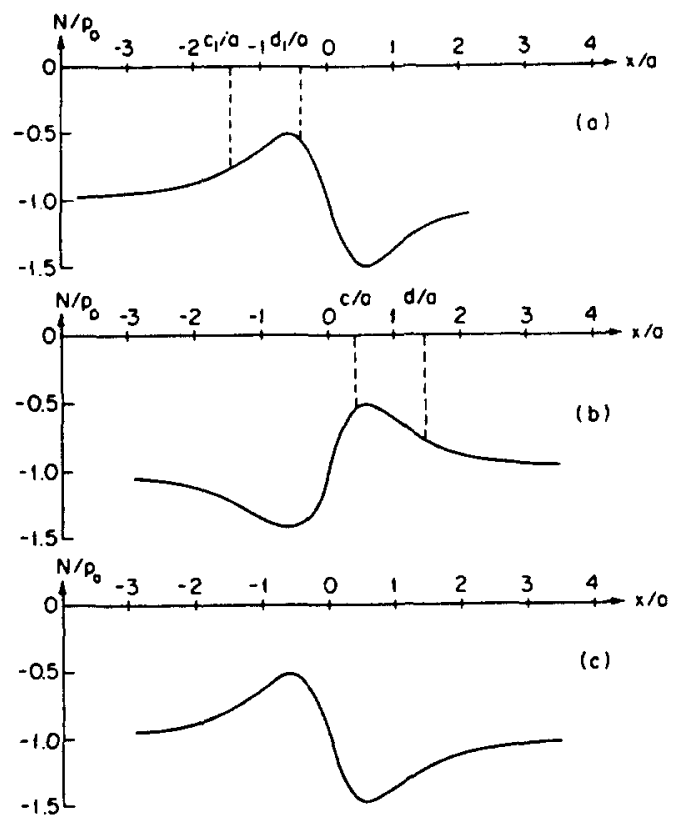

Fig. 4. Normal tractions for $\lambda_{1}=Q_{1} / p_{0} a=2.353$. (a) loading (b) unloading (c) reloading, corresponding to the points $A, B, C$, respectively in Fig. 3 .
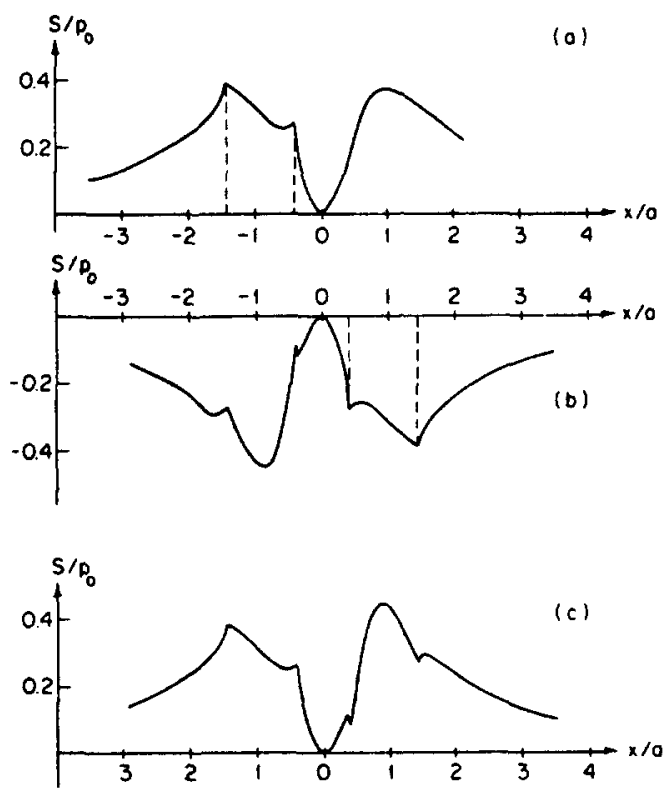

Fig. 5. Shear tractions for $\lambda_{1}=Q_{1} / p_{0} a=2.353$. (a) loading (b) unloading (c) reloading, corresponding to the points $A, B, C$, respectively in Fig. 3 .

This sequence of events-characterized by forward slip on loading, reverse slip in an approximately symmetric zone on unloading, and complete stick in subsequent cycles-was also found for other values of load. It ceases to apply when the cyclic load is large enough to cause backslip in the original slip zone on unloading. Reference to Fig. 5(b) shows that the effect of the locked-in dislocations is unfavorable to preventing slip in this region during unloading and we find that slip occurs if $\lambda_{1}>3.1$.

We can summarize the behavior of the system as follows:

For $\lambda_{1}<2.03$, no slip occurs at any time.

For $3.1>\lambda_{1}>2.03$, slip occurs during loading and unloading in the first complete cycle, but the dislocations locked-in at the slip zones generate a residual stress field which prevents slip in 


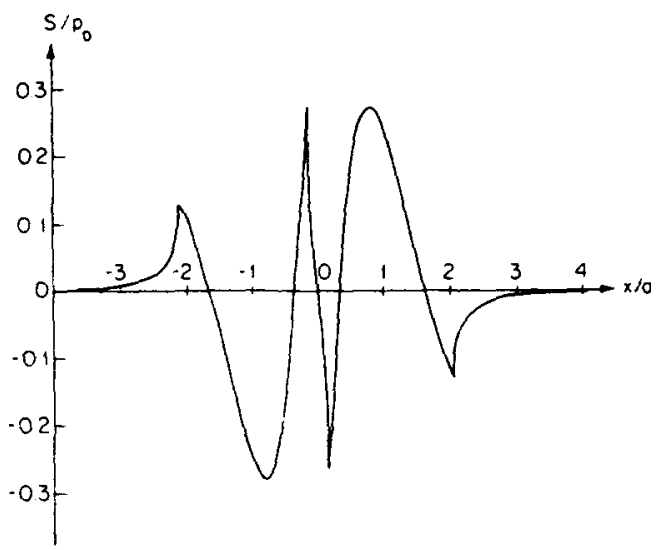

Fig. 6. Residual shear traction at the completion of one cycle close to the shakedown limit, $\lambda_{1}=Q_{1} / p_{0} a=$ 3.1 .

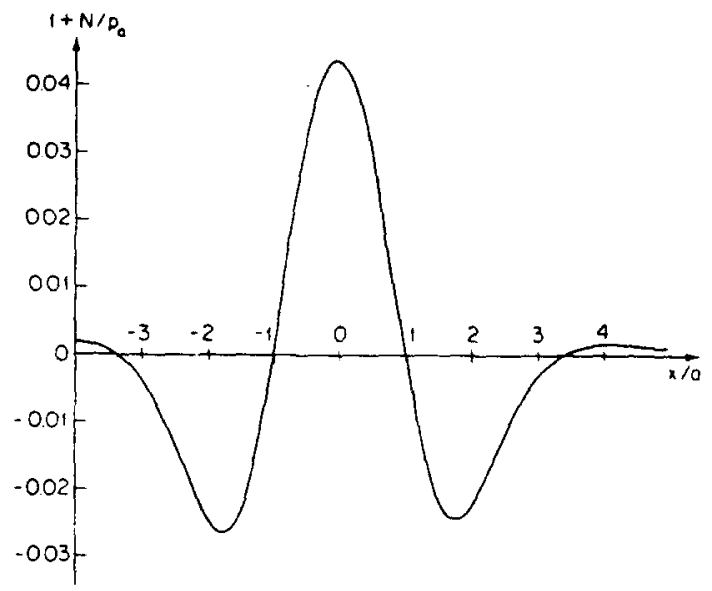

Fig. 7. Residual normal tractions at the completion of one cycle close to the shakedown limit $Q_{1} / p_{0} a=3.1$.

subsequent cycles. These residual tractions (without the uniform compression $p_{0}$ ) are shown in Figs. 6 and 7 for $\lambda_{1}=3.1$. They correspond to the point $D$ in Fig. 3 .

For loads somewhat greater than 3.1, we anticipate that the steady-state will involve slip in every cycle-albeit over smaller regions than those developed in the first cycle. However, this situation is not pursued here, but will form the subject of a further investigation.

It should be noted that all the inequalities of the problem were verified for the solution presented.

Acknowledgement-Support by Office of Naval Research under the contract N00014-81-0626 is gratefully acknowledged.

\section{REFERENCES}

1. M. Comninou, D. Schmueser and J. Dundurs, Frictional slip between a layer and a substrate caused by a normal load. Int. J. Engng Sci. 18, 131-137 (1980).

2. D. Schmueser, M. Comninou and J. Dundurs, Separation and slip between a layer and a substrate caused by a tensile load. Int. J. Engng Sci. 18, 1149-1155 (1980).

3. D. Schmueser, M. Comninou and J. Dundurs, Frictional slip between layet and substrate. J. Engng Mech. Div. ASCE 107, 1103-1118 (1981).

4. R. B. Waterhouse, Fretting Corrosion. Pergamon, New York (1972).

5. P. L. Hurricks, The mechansim of fretting, a review. Wear 15, 389-409 (1970).

6. J. E. Merwin and K. L. Johnson. An analysis of plastic deformation in rolling contact. Proc. Inst. Mech. Engng 177, 676 (1963).

7. S. Timoshenko and J. N. Goodier, Theory of Elasticity. McGraw-Hill, New York (1970).

8. J. Dundurs and $M$. Comninou, Some consequences of the inequality conditions in contact and crack problems. J. Elasticity 107, 71-82 (1979).

9. F. Erdogan and G. Gupta, On the numerical solution of singular integral equations. Quart. Appl. Math. 30, 525-534 (1972). 